\title{
Autoavaliação da memória de jovens universitários
}

\author{
Memory self-assessment of university students
}

\author{
Crislaynne Kelly Carvalho de Melo ${ }^{1}$ (D), Antônio Vitor da Silva Roseno ${ }^{1(0)}$, Arthur José Lopes da Silva ${ }^{2}$ (D),

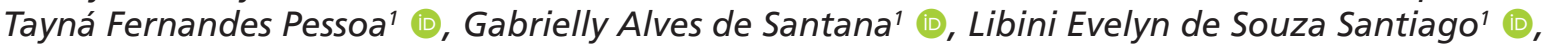 \\ Déborah Lúcia Feitosa Montenegro' (1), Larissa Nadjara Almeida³ (1), Ivonaldo Leidson Barbosa Lima ${ }^{3}$
}

\begin{abstract}
RESUMO
Objetivo: analisar a autoavaliação da memória de jovens universitários. Métodos: trata-se de um estudo observacional, transversal, de caráter analítico, com abordagem quantitativa. Participaram 519 estudantes, matriculados regularmente em instituições de ensino superior, com média de idade de 22,9 anos $( \pm 5,5)$, sendo 408 mulheres e 111 homens. Os instrumentos utilizados foram o Questionário de Memória Prospectiva e Retrospectiva QMPR (Prospective and Retrospective Memory Questionnaire - PRMQ-10) e um questionário com informações sociodemográficas e com queixas de memória relacionadas às atividades acadêmicas. Os dados foram analisados quantitativamente, de forma descritiva e inferencial, com uso do teste Qui-quadrado, considerando o valor de $\mathrm{p}<5 \%$. Resultados: os jovens universitários relataram dificuldades frequentes de memória, em especial na prospectiva e de curto prazo. Foi visto que $46,6 \%$ dos participantes apresentaram queixas de memória e $62,8 \%$ referiram acreditar que a rotina na universidade pode provocar aumento nas falhas de memória. Na autoavaliação, verificou-se que $47,7 \%$ mencionaram dificuldades na memória prospectiva e de curto prazo e, em relação à rotina acadêmica dos universitários, 46,4\% relataram dificuldades na memória retrospectiva. Conclusão: há associação entre a presença de queixa e os dados da autoavaliação da memória.
\end{abstract}

Palavras-chave: Memória; Autoavaliação; Ensino superior; Estudantes; Neuropsicologia; Fonoaudiologia

\begin{abstract}
Purpose: To investigate the memory self-assessment of university students. Methods: Observational, cross-sectional, analytical study with a quantitative approach. A total of 519 students regularly enrolled in higher education institutions participated, with a mean age of 22.9 years $( \pm 5.5)$, being 408 women and 111 men. Data was collected through the Prospective and Retrospective Memory Questionnaire (PRMQ-10) and a questionnaire with sociodemographic information and memory complaints related to academic activities. Data were evaluated quantitatively, descriptively and inferentially, using the Chi-Squared Test with a p-value $<5 \%$. Results: University students reported frequent memory difficulties, especially in the prospective and short term memory. It was found that $46.6 \%$ of the participants had memory complaints and $62.8 \%$ reported believing that routine at the university may lead to an increase in memory failure. In the self-assessment, $47.7 \%$ reported difficulties in prospective and short-term memory and, in relation to the academic routine of university students, $46.4 \%$ reported difficulties in retrospective memory. Conclusion: There is an association between the presence of a memory complaint and the self-assessment of memory data.
\end{abstract}

Keywords: Memory; Self-evaluation; Higher education; Students; Neuropsychology; Speech-Language and Hearing Sciences

Trabalho realizado no Curso de Fonoaudiologia, Centro Universitário de João Pessoa - UNIPÊ - João Pessoa (PB), Brasil.

${ }^{1}$ Curso de Fonoaudiologia, Centro Universitário de João Pessoa - UNIPÊ - João Pessoa (PB), Brasil.

${ }^{2}$ Curso de Ciência da Computação, Universidade Federal da Paraíba - UFPB - João Pessoa (PB), Brasil.

${ }^{3}$ Departamento de Fonoaudiologia, Centro Universitário de João Pessoa - UNIPÊ - João Pessoa (PB), Brasil.

Conflito de interesses: Não.

Contribuição dos autores: $\mathrm{CKCM}$ foi responsável pela concepção e delineamento do estudo, coleta, análise e interpretação dos dados e redação do manuscrito; AVSR, AJLS, TFP, GAS, LESS e DLFM foram responsáveis pela coleta e tabulação dos dados; LNAA foi responsável pela análise e interpretação dos dados e revisão do artigo; ILBL foi responsável pela orientação de todas as etapas do estudo.

Financiamento: Nada a declarar.

Autor correspondente: Ivonaldo Leidson Barbosa Lima. E-mail: ivonaldo.lima@unipe.edu.br

Recebido: Janeiro 26, 2021; Aceito: Junho 06, 2021 


\section{INTRODUÇÃO}

A vida universitária pode ser definida como um período de transformação, no qual ocorrem mudanças e desafios na vida do estudante. A expectativa dessa nova fase de vida demanda um processo adaptativo individual, uma vez que o espaço acadêmico exige uma série de responsabilidades ${ }^{(1)}$.

No Brasil, o número de universitários matriculados em instituições de ensino ultrapassa a casa dos oito milhões, divididos entre 37 mil cursos ofertados por entidades públicas e particulares. De acordo com pesquisas focadas no perfil socioeconômico dos estudantes, observa-se que $54,6 \%$ das vagas são ocupadas por mulheres; referente à média de idade dos universitários, aponta-se para 24,4 anos; $51,2 \%$ dos estudantes são negros ou pardos e $64,7 \%$ concluíram o ensino médio em escolas públicas ${ }^{(2)}$.

As demandas universitárias do sistema de educação brasileiro, em geral, seguem o modelo de aprendizagem tradicional passivo com aulas e provas intercaladas por seminários e estágios curriculares obrigatórios. Sabe-se que o ritmo das aulas e a cobrança social geram estresse no ambiente universitário. Esse estresse, associado a outros fatores pessoais, acaba desenvolvendo ansiedade, depressão ou doenças psicossomáticas. O fator desse estresse está relacionado à competitividade imaginária, gerando uma sobrecarga mental nos estudantes ${ }^{(3)}$.

Além dessas queixas de saúde mental, os universitários também relatam sentir falhas na memória, como dificuldade em memorizar e/ou evocar informações, fator que pode dificultar as atividades acadêmicas diárias desses indivíduos ${ }^{(4)}$.

A memória é o repositório da mente, em que se armazena o que é aprendido e adquirido ${ }^{(5)}$. Sendo assim, é um suporte fundamental para aquisição de novas informações e a prática de qualquer atividade cotidiana que requeira a evocação de conhecimentos aprendidos. Ao armazenar a informação na memória, constrói-se, mentalmente, uma representação do conhecimento e, sem sua existência, não se poderia manejar a aprendizagem e resolver os problemas diários e futuros.

Os estudantes universitários requerem o uso eficiente de vários recursos de memória para executar tarefas cotidianas, como a memorização de determinados conteúdos para escrever em seus suportes, a realização de operações mentais para refletir ou se apropriar de determinado conhecimento e a evocação de informações para realização de provas e seminários. Desse modo, a presença de falhas na memória nos universitários pode prejudicar a realização de várias tarefas que fazem parte da rotina desse grupo.

Estudos indicam que as queixas de memória em jovens têm íntima relação com a ansiedade e o estresse ${ }^{(6-7)}$. Levando em consideração esses dados, torna-se importante pesquisar as queixas de memória em jovens universitários, uma vez que estão mais suscetíveis a comportamentos de estresse e ansiedade, devido à rotina mais frenética, seja ela por conta da demanda da universidade e, para alguns jovens, em razão da sobrecarga com o trabalho.

Sendo assim, é fundamental a investigação das queixas de memórias apresentadas pelos universitários em sua rotina acadêmica, pois a manutenção ou a ampliação dessas dificuldades pode gerar mais sobrecarga e debilitar sua capacidade de raciocínio, memorização, motivação e interesse com relação ao processo de aprendizagem ${ }^{(8)}$.
Identificar e analisar as queixas de memória dos jovens universitários pode contribuir para a identificação dos sistemas de memória mais influenciados pelas demandas do ensino superior e para possibilitar a criação de estratégias direcionadas, para que essas queixas sejam minimizadas e a qualidade da vida acadêmica seja ampliada. Assim, o presente estudo teve como objetivo analisar a autoavaliação da memória de jovens universitários.

\section{MÉTODOS}

Este é um estudo observacional, transversal, de caráter analítico, com abordagem quantitativa, avaliado e aprovado pelo Comitê de Ética em Pesquisas com Seres Humanos do Centro Universitário de João Pessoa - CEP/UNIPÊ, sob protocolo número 3.918.533.

Participaram 519 estudantes matriculados regularmente em instituições de ensino superior, com idade variando entre 18 e 54 anos, média de $22,9( \pm 5,5)$. A amostra foi selecionada por conveniência, de forma não probabilística, atendendo aos seguintes critérios de inclusão: estudantes de ambos os sexos que estavam regulamente matriculados em universidades e tinham acima de 18 anos e abaixo de 54 anos.

Foram excluídos indivíduos que apresentaram alguma comorbidade que, por algum motivo físico ou cognitivo, os incapacitava de compreender e responder aos instrumentos propostos no estudo, ou pudesse interferir nos achados como o diagnóstico de transtornos neurocognitivos ou do neurodesenvolvimento e a realização de tratamento psiquiátrico no momento da pesquisa.

A coleta de dados foi realizada entre abril e maio de 2020, por meio da aplicação de um questionário eletrônico, divulgado por meio de redes sociais. Preliminarmente, os discentes foram informados sobre os objetivos e procedimentos deste estudo, bem como de seus riscos e benefícios. Os que concordaram em participar da pesquisa assinaram o Termo de Consentimento Livre e Esclarecido (TCLE) para que se iniciasse a coleta dos dados.

Em seguida, responderam a um questionário com informações sociodemográficas, como idade, sexo, estado civil, curso, turno do curso, período do curso, atividade laboral diurna/noturna, carga horária de trabalho, presença de queixa de memória, se sofreram algum tipo de lesão cerebral, se tinham histórico de tratamento psiquiátrico e outras informações clínicas.

Posteriormente, os universitários responderam ao Questionário de Memória Prospectiva e Retrospectiva - QMPR (Prospective and Retrospective Memory Questionnaire - PRMQ-10) ${ }^{(9)}$. Esse instrumento foi escolhido por possibilitar a extração de medidas de autoavaliação de diferentes sistemas de memória na população estudada. Ele contempla dez itens referentes à autoavaliação de dificuldades cotidianas de memória de curto e longo prazo, sendo cinco de memória prospectiva e cinco de memória retrospectiva. Cada item é seguido de uma escala Likert de 5 pontos: (1) nunca, (2) raramente, (3) algumas vezes, (4) frequentemente e (5) quase sempre.

Logo após o questionário, os participantes responderam a oito perguntas elaboradas pelos pesquisadores, sendo duas perguntas com resposta "sim" ou "não", a fim de identificar se o estudante acreditava que a sua rotina diária causava falhas na memória e se gostariam de receber informações sobre os cuidados com a memória. As outras seis perguntas verificavam queixas de memória no ambiente acadêmico e contemplavam uma escala de 
Likert de 5 pontos: (1) nunca, (2) raramente, (3) algumas vezes, (4) frequentemente e (5) quase sempre.

Os dados foram tabulados em planilha eletrônica digital para realização de análise estatística descritiva e inferencial. A análise estatística foi realizada por meio do software Statistical Package for Social Sciences (SPSS), versão 20.0.

$\mathrm{Na}$ análise descritiva, foram obtidos dados de média, desvio padrão e frequências relativas e absolutas. Na análise inferencial, foi observada a associação entre a presença de queixa de memória com as dificuldades da autoavaliação da memória, por meio do teste Qui-Quadrado. Em todas as análises estatísticas, foi adotado o nível de significância de 5\%.

\section{RESULTADOS}

No que diz respeito ao perfil sociodemográfico dos estudantes, os resultados mostraram que, dos 519 que participaram da pesquisa, $78,6 \%(n=408)$ eram do sexo feminino, $39,5 \%$ $(n=205)$ estudavam no turno noturno e $14,8 \%(n=77)$ cursavam o $3^{\circ}$ período da graduação. Além disso, $43,5 \%(\mathrm{n}=226)$ dos estudantes trabalhavam durante o dia, 11,6\% $(\mathrm{n}=60)$ possuíam histórico de tratamento psiquiátrico, 46,2\% $(\mathrm{n}=240)$ afirmaram possuir queixa de memória e $62,8 \%(\mathrm{n}=326)$ concordaram que a rotina na universidade provocava falhas em sua memória. De todos os participantes, $91,9 \%(\mathrm{n}=326)$ afirmaram que gostariam de receber informações de cuidados com a memória (Tabela 1).
Em relação aos aspectos observados por meio do PRMQ-10, verificou-se que a maioria dos estudantes relatou ter maior dificuldade nas perguntas que correspondiam à memória prospectiva e memória de curto prazo (Tabela 2). Por exemplo, $47,8 \%(\mathrm{n}=248)$ deles se queixaram de falhas quando decidiam fazer alguma coisa em alguns minutos, mas depois esqueciam de fazer; $43,4 \%(n=225)$ esqueciam alguma coisa que lhes foi dita há alguns minutos; $35,5 \%(n=184)$ não recordavam de levar algo que pretendiam ao sair de casa, mesmo que o objeto estivesse na sua frente e $40,5 \%(n=210)$ dos estudantes esqueciam de dar um recado ou um objeto que lhes pediram que dessem a um visitante (Tabela 2).

Já em relação às dificuldades autorreferidas de memória, referentes à rotina acadêmica, $46,4 \%(\mathrm{n}=241)$ dos estudantes esqueciam com frequência o nome de pessoas próximas; $40,5 \%$ $(n=210)$ relataram que estudavam para as provas, mas na hora de realizar "dava um branco" e 36,6\% ( $n=190)$ esqueciam com frequência o conteúdo discutido em sala de aula (Tabela 3).

Por fim, verificou-se a associação entre a presença de queixa pelos estudantes e a autoavaliação das dificuldades de memória. Pôde-se observar que aqueles que declararam queixa de memória também relataram maior frequência de falhas na autoavaliação do funcionamento mnemônico em situações cotidianas, acadêmicas ou não (Tabela 4).

Tabela 1. Perfil sociodemográfico dos estudantes

\begin{tabular}{|c|c|c|c|}
\hline VARIÁVEIS & & $\mathbf{N}$ & $\%$ \\
\hline \multirow[t]{2}{*}{ Sexo } & Masculino & 111 & 21,4 \\
\hline & Feminino & 408 & 78,6 \\
\hline \multirow[t]{4}{*}{ Turno } & Integral & 145 & 27,9 \\
\hline & Manhã & 92 & 11,7 \\
\hline & Noite & 205 & 39,5 \\
\hline & Tarde & 77 & 14,8 \\
\hline \multirow[t]{10}{*}{ Período } & 1 음 & 59 & 11,4 \\
\hline & $2^{\circ}$ & 43 & 8,3 \\
\hline & $3^{\circ}$ & 77 & 14,8 \\
\hline & $4^{\circ}$ & 37 & 7,1 \\
\hline & 5 & 57 & 11 \\
\hline & $6^{\circ}$ & 52 & 10 \\
\hline & $7^{\circ}$ & 72 & 13,9 \\
\hline & $8^{\circ}$ & 59 & 11,4 \\
\hline & $9^{\circ}$ & 37 & 7,1 \\
\hline & 10 & 26 & 5 \\
\hline \multirow[t]{2}{*}{ Trabalha durante o dia } & Não & 293 & 56,5 \\
\hline & Sim & 226 & 43,5 \\
\hline \multirow[t]{2}{*}{ Trabalha durante a noite } & Não & 481 & 92,7 \\
\hline & Sim & 38 & 7,3 \\
\hline \multirow[t]{2}{*}{ Histórico de tratamento psiquiátrico } & Não & 459 & 88,4 \\
\hline & Sim & 60 & 11,6 \\
\hline \multirow[t]{2}{*}{ Queixa de memória } & Não & 240 & 46,2 \\
\hline & Sim & 279 & 53,8 \\
\hline \multirow{2}{*}{$\begin{array}{l}\text { Você acha que a rotina na universidade provoca um aumento nas falhas } \\
\text { de sua memória? }\end{array}$} & Não & 191 & 36,8 \\
\hline & Sim & 326 & 62,8 \\
\hline \multirow{2}{*}{$\begin{array}{l}\text { Você gostaria de receber mais informações de cuidados com a } \\
\text { memória? }\end{array}$} & Não & 42 & 8,1 \\
\hline & Sim & 477 & 91,9 \\
\hline
\end{tabular}

Legenda: $\mathrm{N}$ = Número de sujeitos; \% = Percentual 
Tabela 2. Autoavaliação - Questionário de Memória Prospectiva e Retrospectiva

\begin{tabular}{|c|c|c|c|c|c|c|c|c|c|c|}
\hline \multirow{2}{*}{ PERGUNTAS } & \multicolumn{2}{|c|}{ Nunca } & \multicolumn{2}{|c|}{ Raramente } & \multicolumn{2}{|c|}{ Às vezes } & \multicolumn{2}{|c|}{ Frequentemente } & \multicolumn{2}{|c|}{$\begin{array}{c}\text { Muito } \\
\text { frequentemente }\end{array}$} \\
\hline & $\mathbf{N}$ & $\%$ & $\mathbf{N}$ & $\%$ & $\mathbf{N}$ & $\%$ & $\mathbf{N}$ & $\%$ & $\mathbf{N}$ & $\%$ \\
\hline $\begin{array}{l}\text { Você decide fazer alguma coisa em alguns minutos e então } \\
\text { esquece de fazê-la? }\end{array}$ & 3 & 0,6 & 69 & 13,3 & 248 & 47,8 & 129 & 24,9 & 70 & 13,5 \\
\hline $\begin{array}{l}\text { Você falha em reconhecer um lugar que você tenha visitado } \\
\text { antes? }\end{array}$ & 163 & 31,4 & 204 & 39,9 & 101 & 19,5 & 34 & 6,6 & 17 & 3,3 \\
\hline $\begin{array}{l}\text { Você falha em alguma coisa que você deveria fazer poucos } \\
\text { minutos mais tarde mesmo que esteja lá na sua frente, } \\
\text { como tomar um remédio ou apagar o fogo? }\end{array}$ & 69 & 13,3 & 139 & 26,8 & 167 & 32,2 & 98 & 18,9 & 46 & 8,9 \\
\hline $\begin{array}{l}\text { Você esquece alguma coisa que lhe foi dita alguns minutos } \\
\text { antes? }\end{array}$ & 21 & 4,0 & 101 & 19,5 & 225 & 43,4 & 102 & 19,7 & 70 & 13,5 \\
\hline $\begin{array}{l}\text { Você esquece de compromissos se não for lembrado por } \\
\text { outra pessoa ou por um lembrete como um calendário ou } \\
\text { uma agenda? }\end{array}$ & 47 & 9,1 & 162 & 31,2 & 154 & 29,7 & 100 & 19,3 & 56 & 10,8 \\
\hline $\begin{array}{l}\text { Você falha em reconhecer uma personagem num programa } \\
\text { de rádio ou de televisão de uma cena para outra? }\end{array}$ & 208 & 40,1 & 195 & 37,6 & 75 & 14,5 & 25 & 4,8 & 16 & 3,1 \\
\hline $\begin{array}{l}\text { Você falha ao lembrar coisas que aconteceram com você } \\
\text { nos últimos dias? }\end{array}$ & 65 & 12,5 & 136 & 26,2 & 191 & 36,8 & 85 & 16,4 & 42 & 8,1 \\
\hline $\begin{array}{l}\text { Você pretende levar algo com você, antes de deixar uma } \\
\text { sala ou sair para a rua, mas, minutos depois, deixa o que } \\
\text { queria levar para trás, mesmo que esteja lá na sua frente? }\end{array}$ & 46 & 8,9 & 127 & 32,9 & 184 & 35,5 & 96 & 18,5 & 66 & 12,7 \\
\hline $\begin{array}{l}\text { Você esquece de dar um recado ou um objeto que lhe } \\
\text { pediram que desse a um visitante? }\end{array}$ & 42 & 8,1 & 127 & 24,5 & 210 & 40,5 & 88 & 17 & 52 & 10 \\
\hline Você esquece o que você viu na televisão no dia anterior? & 95 & 18,3 & 185 & 35,6 & 154 & 29,7 & 59 & 11,4 & 26 & 5,0 \\
\hline
\end{tabular}

Legenda: $\mathrm{N}$ = Número de sujeitos; \% = Percentual

Tabela 3. Autoavaliação de dificuldades de memória relacionadas à rotina acadêmica

\begin{tabular}{|c|c|c|c|c|c|c|c|c|c|c|}
\hline \multirow{2}{*}{ PERGUNTAS } & \multicolumn{2}{|c|}{ Nunca } & \multicolumn{2}{|c|}{ Raramente } & \multicolumn{2}{|c|}{ Às vezes } & \multicolumn{2}{|c|}{ Frequentemente } & \multicolumn{2}{|c|}{$\begin{array}{c}\text { Muito } \\
\text { frequentemente }\end{array}$} \\
\hline & $\mathbf{N}$ & $\%$ & $\mathbf{N}$ & $\%$ & $\mathbf{N}$ & $\%$ & $\mathbf{N}$ & $\%$ & $\mathbf{N}$ & $\%$ \\
\hline Esquece com frequência o nome de pessoas próximas? & 241 & 46,4 & 165 & 31,8 & 71 & 13,7 & 26 & 5,0 & 16 & 3,1 \\
\hline $\begin{array}{l}\text { Geralmente, esquece o prazo de entrega das atividades } \\
\text { acadêmicas? }\end{array}$ & 100 & 19,3 & 179 & 34,5 & 136 & 26,2 & 62 & 11,9 & 42 & 8,1 \\
\hline $\begin{array}{l}\text { Não consegue memorizar os horários das disciplinas } \\
\text { acadêmicas? }\end{array}$ & 143 & 27,6 & 151 & 29,1 & 112 & 21,6 & 62 & 11,9 & 51 & 9,8 \\
\hline Esquece as datas de provas? & 196 & 37,8 & 149 & 28,7 & 94 & 18,1 & 55 & 10,6 & 25 & 4,8 \\
\hline $\begin{array}{l}\text { Esquece com frequência o conteúdo discutido em sala } \\
\text { de aula? }\end{array}$ & 23 & 4,4 & 140 & 27,0 & 190 & 36,6 & 106 & 20,4 & 60 & 11,6 \\
\hline Você estuda, mas na hora da prova "dá um branco"? & 18 & 3,5 & 122 & 23,5 & 210 & 40,5 & 95 & 18,3 & 74 & 14,3 \\
\hline
\end{tabular}

Legenda: $\mathrm{N}$ = Número de sujeitos; \% = Percentual

\section{DISCUSSÃO}

Neste estudo, a maioria dos universitários afirmou possuir queixas de memória e concordou que a rotina na universidade pode provocar tais falhas e portanto, gostariam de receber informações de cuidados com a memória.

$\mathrm{O}$ ambiente universitário abrange uma ampla carga de responsabilidades. Assim, o exercício do controle emocional é uma dificuldade para grande parte dos jovens, frente a essa nova ambientação, uma vez que a carga de atividades prioriza posturas que exigem dedicação de mais tempo e foco do estudante, seja na sala de aula, propriamente dita, ou no futuro ambiente de trabalho. Portanto, a carga horária de estudos elevada pode acarretar ansiedade e, consequentemente, provocar as falhas de memória nesse público.

Outras pesquisas concordam com os dados deste estudo, ao pontuarem que as queixas e as falhas de memória são comuns entre jovens universitários, podendo estar associadas à presença de ansiedade e estresse ${ }^{(7,10)}$. Esses aspectos podem impactar de modo negativo os processos cognitivos dos universitários, causando dificuldade de memorização e evocação. Além disso, a ansiedade pode aumentar o índice de queixas na memória prospectiva e retrospectiva ${ }^{(11)}$, dado evidenciado neste estudo, em que os participantes referiram possuir diferentes dificuldades de memória, em especial, na memória prospectiva e de curto prazo.

Sabe-se que a memória prospectiva é definida como a habilidade da recordação de executar ações futuras, que está presente nas realizações de atividades diárias do indivíduo e o seu funcionamento envolve as funções executivas ${ }^{(12)}$, como planejamento, memória operacional, controle inibitório e flexibilidade cognitiva, que possibilitam ao indivíduo direcionar seus comportamentos para tais objetivos e atingir um bom desempenho nas atividades futuras.

$\mathrm{O}$ ambiente acadêmico exige o pleno funcionamento dessas funções, as quais permitem ao estudante a habilidade de 
Tabela 4. Associação entre a autoavaliação e presença de queixa de memória

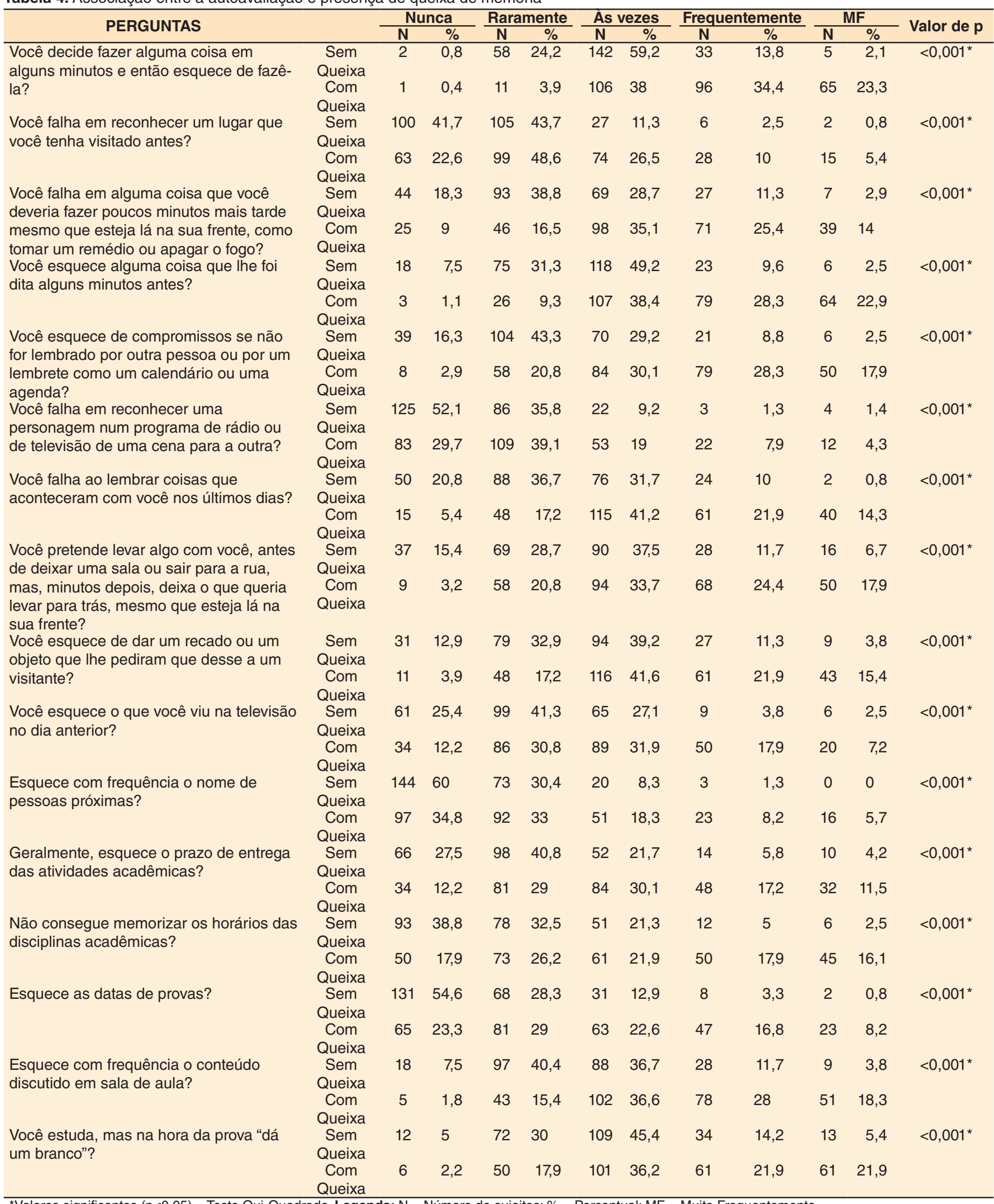

*Valores significantes ( $\mathrm{p} \leq 0,05)$ - Teste Qui-Quadrado. Legenda: N = Número de sujeitos; \% = Percentual; MF = Muito Frequentemente

planejar e realizar tarefas, pensar, reter, manipular e memorizar informações. Diante disso, prejuízos nessas habilidades podem acarretar dificuldades para adquirir novas habilidades, raciocinar, se concentrar e memorizar novas informações ${ }^{(8)}$.
Nas atividades acadêmicas, os universitários apresentaram queixas na memória retrospectiva e de curto prazo. A memória retrospectiva refere-se a recordações de eventos passados e a memória de curto prazo está relacionada com o tempo 
de duração da informação, ou seja, a menor capacidade de armazenamento por um curto período, numa escala de duração de minutos a horas ${ }^{(13)}$.

As informações armazenadas na memória de curto prazo desaparecem rápido ou se transformam em memória de longo prazo. A memória de longo prazo tem a capacidade de armazenamento ilimitada, ou seja, a informação pode permanecer no sistema durante dias, semanas ou até anos ${ }^{(14)}$. Diante disso, a memória tem grande valor na rotina dos jovens universitários, atuando como um importante recurso cognitivo na aprendizagem e os auxiliando nas realizações das práticas diárias ${ }^{(15)}$. Portanto, dificuldades nessas memórias podem comprometer o desempenho esperado nas atividades acadêmicas, como o esquecimento dos conteúdos abordados em sala de aula e, ao estudar o conteúdo, "dar um branco" na hora da prova.

Investigou-se, ainda, a associação entre a presença de queixa e a autoavaliação das falhas de memória. Constatou-se que os estudantes que sinalizaram a presença de queixas de memória no questionário de informações sociodemográficas apontaram maior frequência de falhas na memória em seu cotidiano acadêmico, ou não.

Esse dado evidencia que a autoavaliação cognitiva é um instrumento relevante e que pode ser utilizado para rastreio e monitoramento no âmbito universitário. Um dos sinais iniciais do comprometimento cognitivo da memória é o esquecimento de eventos recentes e a repetição da mesma pergunta ou relato, várias vezes. Desse modo, devem ser levadas em consideração quaisquer alterações sentidas referentes à memória, pois tais falhas podem indicar prejuízos cognitivos associados, ou não, a fatores psicológicos, como ansiedade, depressão e estresse ${ }^{(16-18)}$.

Caso o universitário minimize suas queixas e dificuldades de memória, seu desempenho acadêmico pode sofrer uma influência negativa e, se tais dificuldades forem observadas tardiamente, poderão ocorrer prejuízos na vida cotidiana, impossibilitando a realização de suas atividades com êxito. Assim, vale salientar a importância do desenvolvimento de estratégias que contribuam para a identificação dessas queixas pelos universitários e para adoção dos cuidados devidos na prevenção de falhas de memória.

Em relação aos cuidados com a memória, devem ser elencadas práticas que podem auxiliar os universitários a prevenir e a minimizar as queixas e falhas nesse sistema. Uma delas é a prática de atividades físicas, pois os indivíduos que as praticam regularmente são menos vulneráveis a um declínio cognitivo, quando comparados àqueles não ativos nessa prática ${ }^{(19)}$. Além disso, é importante manter uma dieta saudável com frutas e vegetais $^{(20)}$ e sono regularizado, para que, dessa forma, ocorra efetivamente a consolidação da memória ${ }^{(21)}$.

Concordando com essa perspectiva, um estudo objetivou analisar a memória e a capacidade funcional de idosos que praticavam atividades físicas e de idosos que não realizavam essa prática. Foi possível observar que os idosos praticantes apresentaram melhor desempenho na memória, quando comparados aos idosos não praticantes ${ }^{(22)}$.

Outra intervenção que pode ser realizada é o treino cognitivo computadorizado, que tem o objetivo de aprimorar e prevenir o declínio cognitivo, atividade que pode ser realizada online, com o auxílio de softwares, de forma intensiva e com ajuste do nível de complexidade ${ }^{(23)}$. Estudo ${ }^{(24)}$ realizou um treinamento cognitivo computadorizado em idosos, por um período de oito a dez semanas, com o objetivo de melhorar as habilidades cognitivas; ao fim do treinamento, foi possível verificar o melhor desempenho nas habilidades de memória, atenção auditiva e velocidade de processamento.

Uma estratégia bastante utilizada é o uso de recursos lúdicos para estimulação cognitiva e potencialização da aprendizagem, como os jogos de memória, raciocínio, atenção, caça-palavras, entre outros, pois jogar exige concentração, memória, estratégia, trabalho em equipe e foco, para que se alcance objetivos ${ }^{(25)}$. Ressalta-se, ainda, que a leitura é considerada uma condição básica para o bom funcionamento da memória, pois melhora a percepção visual e auditiva, além de expandir a capacidade da mente, estimulando a criatividade ${ }^{(26)}$.

Além disso, a realização de intervenções clínicas contribui para o melhor funcionamento dos aspectos cognitivos ${ }^{(27)}$. Nessa perspectiva, pode ser exercido um trabalho interdisciplinar com fonoaudiólogo e psicólogo, com o objetivo de realizar a promoção de aspectos cognitivo-linguísticos, a prevenção de possíveis alterações, o melhor desempenho cognitivo e, consequentemente, o aprimoramento da funcionalidade na vida diária do universitário.

Portanto, a intervenção e a estimulação das habilidades cognitivas podem ser aliadas à vida dos universitários e poderiam ser realizadas no próprio ambiente acadêmico, por meio de espaços de promoção da saúde cognitiva, visando contribuir para a adaptação do estudante à vida acadêmica, melhorar seu desenvolvimento, auxiliando sua saúde mental.

Diante disso, o fonoaudiólogo é um dos profissionais capacitados para intervir nesses aspectos, desenvolvendo ações que favoreçam a estimulação e a eliminação das queixas e dificuldades desse público, objetivando contribuir para o bom funcionamento cognitivo do universitário na vida acadêmica.

\section{CONCLUSÃO}

Queixas de memória são frequentes entre os jovens universitários, sendo as mais relatadas as dificuldades na memória prospectiva e de curto prazo. Há associação entre a presença de queixa e os dados da autoavaliação da memória.

A memória exerce um papel importante no contexto acadêmico e os universitários acreditam que a própria rotina da universidade impacta as falhas de memória que apresentam. Portanto, é crucial o desenvolvimento de estratégias para a promoção da saúde cognitiva no ensino superior e para a identificação precoce de falhas mnemônicas.

\section{REFERÊNCIAS}

1. Oliveira CT, Santos AS, Dias ACG. Expectativas de universitários sobre a universidade: sugestões para facilitar a adaptação acadêmica. Rev Bras Orientaç Prof. 2016;17(1):43-53.

2. Brasil. Instituto Nacional de Estudos e Pesquisas Educacionais Anísio Teixeira. Ministério da Educação. Censo da educação superior 2018: Notas estatísticas. Inep/MEC [Internet]; Brasília; 2018 [citado em 2021 Jan 26]. Disponível em: http://download.inep.gov.br/educacao_superior/ censo_superior/documentos/2019/censo_da_educacao_superior_2018notas_estatisticas.pdf

3. Monteiro CFS, Freitas JFM, Ribeiro AAP. Estresse no cotidiano acadêmico: o olhar dos alunos de enfermagem da Universidade Federal do Piauí. Esc Anna Nery Rev Enferm. 2007;11(1):66-72. http://dx.doi. org/10.1590/S1414-81452007000100009. 
4. Beyer C, Staunton C, Moodley K. The implications of methylphenidate use by healthy medical students and doctors in South Africa. BMC Med Ethics. 2014 Mar 4;15(1):20. http://dx.doi.org/10.1186/14726939-15-20. PMid:24592964.

5. Izquierdo I. Memória. 3. ed. Porto Alegre: Artmed; 2018.

6. Corbett B, Weinberg L, Duarte A. The effect of mild acute stress during memory consolidation on emotional recognition memory. Neurobiol Learn Mem. 2017;145:34-44. http://dx.doi.org/10.1016/j. nlm.2017.08.005. PMid:28838881.

7. Molina-Rodriguez S, Pellicer-Porcar O, Mirete-Fructuoso M, MartinezAmoros E. Subjective memory complaints, perceived stress and coping strategies in young adults. Rev Neurol. 2016;62(8):344-50. PMid:27064913.

8. León CBR, Dias NM, Martins GLL, Seabra AG. Executive functions in preschool children: development and relationships with language and behavior. Psicol Teor Prat. 2018;20(3):121-37. http://dx.doi. org/10.5935/1980-6906/psicologia.v20n3p121-137.

9. Benites D, Gomes WB. Tradução, adaptação e validação preliminar do Questionário de Memória Prospectiva e Retrospectiva (PRMQ). Psico-USF. 2007;12(1):45-54. http://dx.doi.org/10.1590/S141382712007000100006 .

10. Nunes TC. Investigação sobre queixas de memória e transtornos associados em acadêmicos de medicina da Universidade Federal Fluminense [dissertação]. Rio de Janeiro: Universidade Federal Fluminense; 2016.

11. Bezerra IAO. Queixas subjetivas de memória e desempenho cognitivo em adultos acima de 50 anos [dissertação]. João Pessoa: Universidade Federal da Paraíba; 2016.

12. Fronda G, Monti C, Sozzi M, Corbo M, Balconi M. Prospective memory and working memory in comparison. New experimental paradigms. Int J Neurosci. 2020;130(8):834-40. http://dx.doi.org/10 $.1080 / 00207454.2019 .1707821$. PMid:31858863.

13. Silva JCM. Evidências de validade do questionário de memória prospectiva e retrospectiva formato pictórico para crianças: PRMQ-P [dissertação]. Maceió: Universidade Federal de Alagoas, Instituto de Psicologia; 2015.

14. Camina E, Güell F. The neuroanatomical, neurophysiological and psychological basis of memory: current models and their origins. Front Pharmacol. 2017;8:438. http://dx.doi.org/10.3389/fphar.2017.00438. PMid:28713278.

15. Mourao CAJr, Faria NC. Memória. Psicol Reflex Crit. 2015;28(4):780-8.

16. Bernardes FR, Machado CK, Souza MC, Machado MJ, Belaunde AMA. Queixa subjetiva de memória e a relação com a fluência verbal em idosos ativos. CoDAS. 2017;29(3):e20160109. http://dx.doi. org/10.1590/2317-1782/20172016109. PMid:28538830.
17. Dalpubel D, Rossi PG, Almeida ML, Ribeiro EB, Araújo R, Andrade LP, et al. Subjective memory complaint and its relationship with cognitive changes and physical vulnerability of community-dwelling older adults. Dement Neuropsychol. 2019;13(3):343-9. http://dx.doi. org/10.1590/1980-57642018dn13-030012. PMid:31555408.

18. Balash Y, Mordechovich M, Shabtai H, Giladi N, Gurevich T, Korczyn AD. Subjective memory complaints in elders: depression, anxiety, or cognitive decline? Acta Neurol Scand. 2013;127(5):344-50. http:// dx.doi.org/10.1111/ane.12038. PMid:23215819.

19. Scianni AA, Faria GS, Silva JS, Benfica PA, Faria CDCM. Efeitos do exercício físico no sistema nervoso do indivíduo idoso e suas consequências funcionais. Rev Bras Ciênc Esporte. 2019;41(1):81-95. http://dx.doi.org/10.1016/j.rbce.2018.03.026.

20. Reichelt AC, Westbrook FR, Morris MJ. Editorial: impact of diet on learning, memory and cognition. Front Behav Neurosci. 2017;11:96. http://dx.doi.org/10.3389/fnbeh.2017.00096. PMid:28579950.

21. Santos TCMM, Martino MMF, Sonati JC, Faria AL, Nascimento EFA. Qualidade do sono e cronotipo de estudantes de enfermagem. Acta Paul Enferm. 2016;29(6):658-63. http://dx.doi.org/10.1590/19820194201600092.

22. Cordeiro J, Del Castillo BL, Freitas CS, Gonçalves MP. Efeitos da atividade física na memória declarativa, capacidade funcional e qualidade de vida em idosos. Rev Bras Geriatr Gerontol. 2014;17(3):541-52. http://dx.doi.org/10.1590/1809-9823.2014.13006.

23. Cardoso CO, Dias NM, Seabra AG, Fonseca RP. Program of neuropsychological stimulation of cognition in students: emphasis on executive functions - development and evidence of content validity. Dement Neuropsychol. 2017;11(1):88-99. http://dx.doi. org/10.1590/1980-57642016dn11-010013. PMid:29213498.

24. Smith GE, Housen P, Yaffe K, Ruff R, Kennison RF, Mahncke $\mathrm{HW}$, et al. A cognitive training program based on principles of brain plasticity: results from the Improvement in Memory with Plasticitybased Adaptive Cognitive Training (IMPACT) study. J Am Geriatr Soc. 2009;57(4):594-603. http://dx.doi.org/10.1111/j.1532-5415.2008.02167.x. PMid:19220558.

25. Sandeep S, Shelton CR, Pahor A, Jaeggi SM, Seitz AR. Application of machine learning models for tracking participant skills in cognitive training. Front Psychol. 2020 Jul 22;11:1532. http://dx.doi.org/10.3389/ fpsyg.2020.01532. PMid:32793032.

26. Gabriel R, Morais J, Kolinsky R. A aprendizagem da leitura e suas implicações sobre a memória e a cognição. Ilha Desterro. 2016;69(1):6178. http://dx.doi.org/10.5007/2175-8026.2016v69n1p61.

27. Golino MTS, Flores-Mendoza CE. Desenvolvimento de um programa de treino cognitivo para idosos. Rev Bras Geriatr Gerontol. 2016;19(5):76985. http://dx.doi.org/10.1590/1809-98232016019.150144. 